\title{
Testable implications for the Bresnahan-Lau model of market competition
}

\author{
Laurens Cherchye, Thomas Demuynck ${ }^{\dagger}$ and Bram De Rock ${ }^{\ddagger}$
}

April 2010

\begin{abstract}
We derive necessary and sufficient testable implications on the reduced form price and quantity functions for the Bresnahan-Lau model of market competition. The conditions are twofold. A first condition relates to the fact that the reduced form price function should correspond to an inverse demand function. The second condition captures that the degree of competition must be invariant to changes in the exogenous variables.
\end{abstract}

\section{Introduction}

Motivation The Bresnahan-Lau model (BL-model from now on) of firm competition (Bresnahan, 1982; Lau, 1982) is widely used to empirically analyze the degree of competition within a given market. In this model, the markup of price over marginal cost is related to a parameter that measures the degree to which the firms in the market behave competitively. Following Corts (1999), we call this parameter the conduct parameter. If the conduct parameter is zero, there is no market power and the firms behave in perfect competition. If the parameter equals one, then the firms behave like a monopolist, maximizing the cartel profit. The relevance of measuring the conduct parameter is straightforward. Increased market power and collusive behavior leads to strong negative welfare effects on the demand side of the market. If we are capable of estimating the value of the conduct parameter, then we can -at least in principle- decide whether or not certain firms abuse their market power.

As a theoretical construct, a firm's conduct parameter is usually interpreted as the change in aggregate output in response to an infinitesimal increase in the output of this firm (i.e. conjectural variation). Although this interpretation is controversial from a theoretical point of view, ${ }^{1}$ the BLmodel remains widely employed in the New Empirical Industrial Organization literature until this day. $^{2}$ Indeed, an attractive property of the BL-model is that it provides an easily implemented set of conditions that are sufficient to establish econometric identification. Focusing on a linear

${ }^{*}$ CentER, Tilburg University and Center for Economic Studies, University of Leuven. E. Sabbelaan 53, B8500 Kortrijk, Belgium. email: L.Cherchye@uvt.nl and laurens.cherchye@kuleuven-kortrijk.be. Laurens Cherchye gratefully acknowledges financial support from the Research Fund K.U.Leuven through the grant STRT1/08/004.

${ }^{\dagger}$ Center for Economic Studies, University of Leuven, and Sherppa, Ghent University. E. Sabbelaan 53, B-8500 Kortrijk, Belgium. email: thomas.demuynck@kuleuven-kortrijk.be. Thomas Demuyunck gratefully acknowledges the Fund for Scientific Research Flanders (FWO-Vlaanderen) for his postdoctoral fellowship.

${ }^{\ddagger}$ ECARES-ECORE, Université Libre de Bruxelles. Avenue F.D. Roosevelt 50, CP 114, B-1050 Brussels, Belgium. email: bderock@ulb.ac.be

${ }^{1}$ However, see d'Aspremont, Dos Santos Ferreira, and Gérard-Varet (2007), and d'Aspremont and Dos Santos Ferreira (2009) who provide several rationales for this conduct parameter using a game theoretic approach.

${ }^{2}$ See, among many others, Zeidan (2009); Coccorese (2008); Durevall (2007); Coccorese (2005); Delis, Staikouras, and Varlagas (2008); Bihari and Seldon (2006); Fisher and Kamerschen (2003); Toolsema (2002); Shaffer (2001); Raper, Love, and Shumway (2000); Neven and Roller (1999); Steen and Salvanes (1999); Kadiyali, Vilcassim, and Chintagunta (1999); Nevo (1998); Chulho (1995); Nebesky, McMullen, and Lee (1995); Graddy (1995); Berg and Kim (1994); Seldon, Banerjee, and Boyd (1993); Rubinovitz (1993); Claycombe and Mahan (1993); Suzuki, Lenz, and Forker (1993); Brander and Zhang (1990). See also Bresnahan (1989) for a somewhat dated but extensive overview of the literature before 1990) 
demand function, Bresnahan (1982) showed that identification is guaranteed if one introduces a rotation variable in the aggregate demand equation, i.e. it suffices to introduce an exogenous variable that shifts the slope of the demand function. Lau (1982) extended this result by showing identification even without assuming a particular functional structure for the reduced form price and quantity function. He finds that the conduct parameter is identified as long as aggregate demand is non-separable in at least one exogenous variable.

Empirical applications of the BL-model usually proceed in four steps. In a first step, one imposes a functional form on the inverse demand and marginal cost function in terms of output, exogenous demand shifters and marginal cost shifters (while -of course- accounting for the identification restrictions). In a second step, one derives the supply equations (by using the first order condition (1) below). In a third step, both demand and supply equations are estimated, while taking into account various endogeneity issues. Finally, the conduct parameter is retrieved as a function of the estimated parameters.

Contribution Despite its wide use, empirical applications of the BL-model have used this framework without verifying its strong underlying assumptions, such as the existence of the inverse demand function and/or the existence of a constant conduct parameter. Although the above procedure gives an estimate of the conduct parameter in cases where the true model satisfies the presupposed functional specifications, it does not give any guidance with respect to the question whether the BL-model corresponds to the true underlying data generating process. This note fills this gap by providing necessary and sufficient conditions on the reduced form price and quantity functions such that these are consistent with an inverse demand function and a marginal cost function for some underlying value of the conduct parameter. As in Lau (1982), our analysis will be nonparametric in that we abstain from imposing any functional specification for the demand function or the marginal cost function. As a by-product, we derive a closed form solution (in terms of the reduced form price and quantity functions) for the conduct parameter, whenever identified.

\section{Outline of the BL-model}

To set the stage, we provide an outline of the BL-model. In doing so, we will also introduce necessary notation, definitions and assumptions.

Let $P(Q, z)$ be the inverse demand function for the output of an industry, with $Q$ the quantity of the output and $z=\left(z^{1}, \ldots, z^{n}\right)$ a vector of $n$ exogenous variables that affect the industry demand but not the marginal cost of the firms. Further, let $M C(Q, w)$ be the marginal cost function with $w=\left(w^{1}, \ldots, w^{m}\right)$ a vector of $m$ exogenous variables that affect the marginal cost function but not the industry demand. We assume that $(z, w) \in \mathcal{O} \subseteq \mathbb{R}^{n+m}$, with $\mathcal{O}$ an open and connected set.

The BL-model of industry competition assumes the existence of a fixed parameter $\lambda$ such that the equilibrium quantity $Q^{*}$ satisfies the following first order condition:

$$
P\left(Q^{*}, z\right)+\lambda \frac{\partial P\left(Q^{*}, z\right)}{\partial Q} Q^{*}=M C\left(Q^{*}, w\right) .
$$

Obviously, the value of $Q^{*}$ will be a function of the exogenous variables $z$ and $w$. The conduct parameter $\lambda$ takes values between zero and unity; it measures the degree of competitiveness of the industry. For $\lambda=0$, one obtains:

$$
P\left(Q^{*}, z\right)=M C\left(Q^{*}, w\right),
$$

which gives the first order condition for the model under perfect competition. If $\lambda=1$, one derives:

$$
P\left(Q^{*}, z\right)+\frac{\partial P\left(Q^{*}, z\right)}{\partial Q} Q^{*}=M C\left(Q^{*}, w\right),
$$

which coincides with the first order condition for the model with perfect cartel formation. Bresnahan (1982) and Lau (1982) derived the necessary and sufficient conditions for which the conduct 
parameter is identified. Under these conditions, $\lambda$ can be uniquely determined for sufficient variation in observed equilibrium prices, output, and exogenous variables $z$ and $w$.

In this paper we focus on the related but distinct issue of deriving testable implications of the BL-model. This question is of importance as not every data set is consistent with a constant value for the conduct parameter $\lambda$ or with the existence of an inverse demand function. We use the framework of Lau (1982) and assume that we observe the reduced form price and quantity functions $q(z, w)$ and $p(z, w)$ associated with the exogenous variables $z$ and $w$. We further assume that both functions $p(z, w)$ and $q(z, w)$ are $C^{2}$ on $\mathcal{O}$. The following definition characterizes the set of functions $p(z, w)$ and $q(z, w)$ that are consistent with the BL-model:

Definition 1. The functions $q(z, w)$ and $p(z, w)$ are consistent with the $B L$-model if there exist a (differentiable) inverse demand function $P(Q, z)$, decreasing in $Q$, a (differentiable) marginal cost function $M C(Q, w)$ and a constant $\lambda$ such that for all values of $z, w \in \mathcal{O}$ :

- $P(q(z, w), z)=p(z, w)$, and,

- $P(q(z, w), z)+\lambda \frac{\partial P(q(z, w), z)}{\partial Q} q(z, w)=M C(q(z, w), w)$.

We make the following mild assumption to ensure non-triviality of $q(z, w)$ :

Assumption 1. Take any $(z, w) \in \mathcal{O}$, then there exists at least one $i \leq n$ and one $t \leq m$ such that:

$$
\frac{\partial q(z, w)}{\partial z^{i}} \neq 0 \quad \text { and } \quad \frac{\partial q(z, w)}{\partial w^{t}} \neq 0 .
$$

For example, this assumption is always satisfied if $q(z, w)$ is strictly monotone in one variable $z^{i}$ and one variable $w^{t}$. Clearly, this assumption is verifiable for any given function $q(z, w)$.

\section{Testable implications of the BL-model}

We first derive two necessary conditions for the case where $n, m \geq 2$. Next, we will show that these conditions are also sufficient for the BL-model to hold, and we will relax the constraint on $n$ and $m$. As we will explain, the first condition guarantees the existence of an inverse demand function, while the second condition captures that the conduct parameter must be invariant to changes in the exogenous environment.

The first condition relates $p(z, w)$ to an inverse demand function $P(q(z, w), z)$. It reflects the fact that a change in $w$ only influences $p$ through the quantity function $q(z, w)$. Take any $k, t \leq m$. Then, differentiating the identity $p(z, w)=P(q(z, w), z)$ with respect to $w^{k}$ and $w^{t}$ gives:

$$
\frac{\partial p(z, w)}{\partial w^{k}}=\frac{\partial P(q(z, w), z)}{\partial Q} \frac{\partial q(z, w)}{\partial w^{k}} \quad \text { and } \quad \frac{\partial p(z, w)}{\partial w^{t}}=\frac{\partial P(q(z, w), z)}{\partial Q} \frac{\partial q(z, w)}{\partial w^{t}}
$$

The corresponding testable implication requires, for all $(z, w) \in \mathcal{O}$ and $k, t \leq m$ :

$$
\frac{\partial p(z, w)}{\partial w^{k}} \frac{\partial q(z, w)}{\partial w^{t}}=\frac{\partial p(z, w)}{\partial w^{t}} \frac{\partial q(z, w)}{\partial w^{k}}
$$

Under Assumption 1, (2) allows us to obtain $\tau(z, w) \equiv \frac{\partial P(q(z, w), z)}{\partial Q}$. Specifically, for any $(z, w) \in \mathcal{O}$, there exists at least one $t \leq m$ such that:

$$
\tau(z, w)=\frac{\partial P(q(z, w), z)}{\partial Q}=\frac{\frac{\partial p(z, w)}{\partial w^{t}}}{\frac{\partial q(z, w)}{\partial w^{t}}} .
$$


Note that $\tau(z, w)$ is well defined by Assumption 1 and is independent of $t$ by (A.1). Further, as $P(q(z, w))$ is decreasing in $q, \tau(z, w)$ should be non-positive:

$$
\tau(z, w) \leq 0
$$

The second condition pertains to the marginal cost function $M C(q(z, w), w)$ and the conduct parameter $\lambda$. It uses two features of the BL-model. Firstly, similar to before, changes in $z$ can only influence $M C$ through the function $q(z, w)$. Secondly, the parameter $\lambda$ must be constant for all $(z, w) \in \mathcal{O}$.

More formally, let us consider the first order condition (1) for the BL-model:

$$
P(q(z, w), z)+\lambda \frac{\partial P(q(z, w), z)}{\partial Q} q(z, w)=M C(q(z, w), w) .
$$

Differentiating with respect to $z^{i}$ and $z^{j}, i, j \leq n$, obtains:

$$
\begin{aligned}
{\left[\frac{\partial P(q(z, w), z)}{\partial Q}\right.} & \left.\frac{\partial q(z, w)}{\partial z^{i}}+\frac{\partial P(q(z, w), z)}{\partial z^{i}}\right] \\
& +\lambda\left[\frac{\partial^{2} P(q(z, w), z)}{\partial Q \partial z^{i}} q(z, w)+\frac{\partial^{2} P(q(z, w), z)}{\partial Q^{2}} \frac{\partial q(z, w)}{\partial z^{i}} q(z, w)+\frac{\partial P(q(z, w), z)}{\partial Q} \frac{\partial q(z, w)}{\partial z^{i}}\right] \\
& =\frac{\partial M C(q(z, w), w)}{\partial Q} \frac{\partial q(z, w)}{\partial z^{i}}
\end{aligned}
$$

and

$$
\begin{aligned}
{\left[\frac{\partial P(q(z, w), z)}{\partial Q}\right.} & \left.\frac{\partial q(z, w)}{\partial z^{j}}+\frac{\partial P(q(z, w), z)}{\partial z^{j}}\right] \\
& +\lambda\left[\frac{\partial^{2} P(q(z, w), z)}{\partial Q \partial z^{j}} q(z, w)+\frac{\partial^{2} P(q(z, w), z)}{\partial Q^{2}} \frac{\partial q(z, w)}{\partial z^{j}} q(z, w)+\frac{\partial P(q(z, w), z)}{\partial Q} \frac{\partial q(z, w)}{\partial z^{j}}\right] \\
& =\frac{\partial M C(q(z, w), z)}{\partial Q} \frac{\partial q(z, w)}{\partial z^{j}} .
\end{aligned}
$$

Multiplying equation (4) by $\frac{\partial q(z, w)}{\partial z^{j}}$ and equation (5) by $\frac{\partial q(z, w)}{\partial z^{i}}$ leads to the following condition:

$$
\lambda q(z, w)\left[\frac{\partial^{2} P(q(z, w), z)}{\partial Q \partial z^{i}} \frac{\partial q(z, w)}{\partial z^{j}}-\frac{\partial^{2} P(q(z, w), z)}{\partial Q \partial z^{j}} \frac{\partial q(z, w)}{\partial z^{i}}\right]
$$

Or, equivalently,

$$
\begin{aligned}
\lambda q(z, w)\left[\frac{\partial q(z, w)}{\partial z^{j}} \frac{\partial \tau(z, w)}{\partial z^{i}}\right. & \left.-\frac{\partial q(z, w)}{\partial z^{i}} \frac{\partial \tau(z, w)}{\partial z^{j}}\right] \\
& =\left[\frac{\partial q(z, w)}{\partial z^{i}} \frac{\partial p(z, w)}{\partial z^{j}}-\frac{\partial q(z, w)}{\partial z^{j}} \frac{\partial p(z, w)}{\partial z^{i}}\right] .
\end{aligned}
$$

Thus, the BL-model holds only if there exists a constant conduct parameter $\lambda$ such that equation (B) is satisfied for all $i, j \leq n$ and $(z, w) \in \mathcal{O}$.

Our main result states that the necessary conditions (A.1), (A.2) and (B) are also sufficient for the BL-model to hold:

Theorem 1. Consider two functions $p(z, w)$ and $q(z, w)$ that are $C^{2}$ on $\mathcal{O}$ and that satisfy Assumption 1. Then, there exists a function $P(Q, z)$, which is $C^{2}$ and decreasing in $Q$, a marginal cost function $M C(Q, w)$, which is $C^{2}$, and a constant $\lambda$ such that for all $(z, w) \in \mathcal{O}$ : 
- $P(q(z, w), z)=p(z, w)$, and,

- $P(q(z, w), z)+\lambda \frac{\partial P(q(z, w), z)}{\partial Q} q(z, w)=M C(q(z, w), w)$.

if and only if

A.1) for all $k, t \leq m$ and all $(z, w) \in \mathcal{O}$ :

$$
\frac{\partial p(z, w)}{\partial w^{k}} \frac{\partial q(z, w)}{\partial w^{t}}=\frac{\partial p(z, w)}{\partial w^{t}} \frac{\partial q(z, w)}{\partial w^{k}} ;
$$

A.2) for any $(z, w) \in \mathcal{O}$ and for all $t \leq m$ that satisfy the inequality condition in Assumption 1:

$$
\frac{\frac{\partial p(z, w)}{\partial w^{t}}}{\frac{\partial q(z, w)}{\partial w^{t}}} \equiv \tau(z, w) \leq 0 ;
$$

B) there exist a constant $\lambda \in[0,1]$ such that for all $i, j \leq n$ and all $(z, w) \in \mathcal{O}$ :

$$
\begin{aligned}
& \lambda q(z, w)\left[\frac{\partial q(z, w)}{\partial z^{j}} \frac{\partial \tau(z, w)}{\partial z^{i}}-\frac{\partial q(z, w)}{\partial z^{i}} \frac{\partial \tau(z, w)}{\partial z^{j}}\right] \\
& =\left[\frac{\partial q(z, w)}{\partial z^{i}} \frac{\partial p(z, w)}{\partial z^{j}}-\frac{\partial q(z, w)}{\partial z^{j}} \frac{\partial p(z, w)}{\partial z^{i}}\right] .
\end{aligned}
$$

Proof. Necessity for $n, m \geq 2$ was demonstrated above, so here we restrict ourselves to sufficiency for this case. Our proof relies to a large extent on a lemma of Goldman and Uzawa (1964):

Lemma 1. Consider two continuously differentiable (path connected) functions $f(x)$ and $g(x)$, with $x \in \mathbb{R}^{n}$. Then, if there exists a function $\eta$ such that for all $x$ :

$$
\frac{\partial f(x)}{\partial x^{i}}=\eta(x) \frac{\partial g(x)}{\partial x^{i}} \quad \forall i \leq n,
$$

then there exist a function $F$ such that:

$$
f(x)=F(g(x)) .
$$

Condition (A.1) implies that $\frac{\partial p(z, w)}{\partial w^{k}}=0$ if $\frac{\partial q(z, w)}{\partial w^{k}}=0$ (for some $t$ in (A.1) that satisfies the condition in Assumption 1). Thus, we have that conditions (A.1) and (A.2) imply

$$
\frac{\partial p(z, w)}{\partial w^{k}}=\tau(z, w) \frac{\partial q(z, w)}{\partial w^{k}} \quad \forall k \leq m .
$$

Then Lemma 1 states there for any $z$ exist a function $P$ such that $p(z, w)=P(q(z, w), z)$. Given that $p(z, w)$ and $q(z, w)$ are $C^{2}$, a similar reasoning as in equation (2) shows that $P(Q, z)$ is also $C^{2}$. Finally, by condition (A.2) this function is decreasing in its first argument.

Next, let condition (B) define $\lambda$, and consider the following function $g(z, w)$ :

$$
g(z, w)=p(z, w)+\lambda \tau(z, w) q(z, w) .
$$

One can easily verify that condition (B) implies $\frac{\partial g(z, w)}{\partial z^{i}} \frac{\partial q(z, w)}{\partial z^{j}}=\frac{\partial g(z, w)}{\partial z^{j}} \frac{\partial q(z, w)}{\partial z^{i}}$ for all $i, j \leq$ $n$. Now take any $(z, w) \in \mathcal{O}$ and assume $i \leq n$ satisfies the inequality condition in Assumption 1. Then, we can define

$$
\delta(z, w)=\frac{\frac{\partial g(z, w)}{\partial z^{i}}}{\frac{\partial q(z, w)}{\partial z^{i}}} .
$$


In turn, this yields

$$
\frac{\partial g(z, w)}{\partial z^{i}}=\delta(z, w) \frac{\partial q(z, w)}{\partial z^{i}} \quad \forall i \leq n
$$

Similar to before, Lemma 1 implies that there exists a $C^{2}$-function $M C$ such that $g(z, w)=$ $M C(q(z, w), w)$ for all $(z, w)$.

To finish the proof, we still need to consider the case with $n$ and/or $m$ equal to one. If $m=1$, then condition (A.1) is of course redundant and condition (A.2) is equivalent to condition (6). An argument that is readily similar to the one above shows that condition (A.2) is both necesary and sufficient for the BL-model to hold. If $n=1$, then condition (B) is equivalent to (7), which again obtains the wanted results.

\section{References}

Berg, S. A., Kim, M., 1994. Oligopolistic Interdependence and the Structure of Production in Banking: and Empirical Evaluation. Journal of Money, Credit and Banking 26, 309-322.

Bihari, S., Seldon, B. J., 2006. The Effect of Governement Advertising Policies on the Market Power of Cigarette Firms. Review of Industrial Organization 28, 201-229.

Brander, J. A., Zhang, A., 1990. Market Conduct in the Airline Industry: an Empirical Investication. RAND Journal of Economics 21, 567-583.

Bresnahan, T., 1982. The Oligopoly Solution Concept is Identified. Economics Letters 10, 87-92.

Bresnahan, T., 1989. The Handbook of Industrial Organization. Vol. II. Elsevier, Amsterdam, Ch. Empirical Studies of Industries with Market Power.

Chulho, J., 1995. The Degree of Competition in the Advertising Industry. Review of Industrial Organization 10, 41-52.

Claycombe, R. J., Mahan, T. E., 1993. Spatial Aspects of Retail Market Structure Beef Pricing Revisited. International Journal of Industrial Organization 11, 283-291.

Coccorese, P., 2005. Competition in Markets with Dominant Firms: A Note on the Evidence from the Italian Banking Industry. Journal of Banking and Finance 29, 1083-1093.

Coccorese, P., 2008. Bank Competition and Regional Differences. Economics Letters 101, 13-16.

Corts, K. S., 1999. Conduct Parameters and the Measurement of Market Power. Journal of Econometrics $88,227-250$.

d'Aspremont, C., Dos Santos Ferreira, R., 2009. Price-Quantity Competition with Varying Toughness. Games and Economic Behavior 65, 62-82.

d'Aspremont, C., Dos Santos Ferreira, R., Gérard-Varet, L., 2007. Competition for Market Share or for Market Size: Oligopolisitc Equilibria with Varying Competitive Toughness. International Economic Review 48, 761-784.

Delis, M. D., Staikouras, K. C., Varlagas, P. T., 2008. On the Measurement of Market Power in the Banking Industry. Journal of Business Finanace and Accounting 35, 1023-1047.

Durevall, D., 2007. Competition in the Swedish Coffee Market. International Journal of Industrial Organization 25, 1978-2002.

Fisher, T., Kamerschen, D. R., 2003. Price-Cost Margins in the US airline Industry using a Conjectural Variation Apporach. Journal of Transport Economics and Policy 37, 227-259. 
Goldman, S. M., Uzawa, H., 1964. A Note on Separability in Demand Analysis. Econometrica 32, 387-398.

Graddy, K., 1995. Testing for Imperfect Competition at the Fulton Fish Market. RAND Journal of Economics 26, 75-92.

Kadiyali, V., Vilcassim, N., Chintagunta, P., 1999. Product Line Extensions and Competitive Market Interactions: an Empirical Analysis. Journal of Econometrics 82, 339-363.

Lau, L., 1982. On Identifying the Degree of Competitiveness from Industry Price and Output Data. Economics Letters 10, 93-99.

Nebesky, W., McMullen, B. S., Lee, M.-K., 1995. Testing for Market Power in the U.S. Motor Carrier Industry. Review of Industrial Organization 10, 559-576.

Neven, D., Roller, L., 1999. An Aggregate Structural Model of Competition in the European Banking Industry. International Journal of Industrial Organization 17, 1059-1074.

Nevo, A., 1998. Identification of the Oligopoly Solution Concept in a Differentiated-Product Industry. Economics Letters 39, 391-395.

Raper, K. C., Love, A., Shumway, R., 2000. Determining Market Power, Extension between Buyers and Sellers. Journal of Applied Econometrics 15, 225-252.

Rubinovitz, R., 1993. Market Power and Price Increase for Basic Cable Service since Deregulation. RAND Journal of Economics 24, 1-18.

Seldon, B. J., Banerjee, S., Boyd, R. G., 1993. Advertising Conjectures and the Nature of Advertising Competition in an Oligopoly. Managerial and Decision Economics 14, 489-498.

Shaffer, S., 2001. Banking Conduct Before the European Single Banking License: a Cross-Country Comparison. The North American Journal of Economics and Finance 12, 79-104.

Steen, F., Salvanes, K. G., 1999. Testing for Market Power using a Dynamic Oligopoly Model. International Journal of Industrial Organization 17, 147-177.

Suzuki, H., Lenz, J., Forker, O., 1993. A Conjectural Variations Model of Reduced Japanese Milk Price Supports. American Journal of Agricultural Economics 75, 210-218.

Toolsema, L. A., 2002. Competition in the Dutch Consumer Credit Market. Journal of Banking and Finance 26, 2215-2229.

Zeidan, R. M., 2009. Measuring Market Conduct in the Brazilian Cement Industry: A Dynamic Econometric Investigation. Review of Industrial Organization 34, 231-244. 